\title{
A Multi-stages Decision Approach for Managerial Flexibility of Energy R\&D Project under Fuzzy Environment
}

\author{
Changsheng $\mathrm{Yi}^{1}{ }^{\&}$ Qiumei $\mathrm{Jin}^{2}$ \\ ${ }^{1}$ School of Management Science and Engineering, Anhui University of Technology, Ma'anshan, China \\ 2 The Hospital of Anhui University of Technology, Ma'anshan, China \\ Correspondence: Changsheng Yi, School of Management Science and Engineering, Anhui University of Technol- \\ ogy, Ma'anshan, China. E-mail: yics2005@163.com
}

Received: July 1, 2015 Accepted: July 10, 2015 Online Published: July 24, 2015

doi:10.5539/ijsp.v4n3p169 URL: http://dx.doi.org/10.5539/ijsp.v4n3p169

The research is financed by a grant from the Humanities and Social Sciences Foundation of the Ministry of Education (10YJC630352) and a grant from the Humanities and Social Sciences Major Program of Anhui Province (SK2014ZD018).

\begin{abstract}
In recent years, many countries and firms seek the new and renewable energy to cope with the impending global environmental crisis, such as depletion of fossil-based energy, climate change to control emissions of greenhouse gases. This paper aims to take the perspective of the firm, which undertakes the energy R\&D project to maximize profits implying minimization of total cost as well. Incorporating technical and market risks into energy R\&D project is crucial, in that the managers often face the rapidly changing environment full of uncertainties. The firms should incorporate managerial flexibility into energy R\&D project decision not only reducing uncertain risks, but also increasing potential market payoff. This research considers a multi-stages decision model in which real-option-based analysis is applied for energy R\&D project under fuzzy environment. Specifically, the market payoff is obtained when the new and renewable energy product is commercialized to market, while energy R\&D investment costs are exhausted gradually. Furthermore, the uncertain development performance and market information are described as fuzzy variables by credibility theory. Instead of the traditional real option pricing methods, the dynamic programming methodology that captures the uncertain product development performance and final market return is developed to more effectively characterize the managerial flexibility. This method can reflect the multi-stages nature of R\&D programme, while helping decision-makers take the optimal investment decision and capture future market opportunities of energy products.
\end{abstract}

Keywords: Energy R\&D project, managerial flexibility, fuzzy variable, real option

\section{Introduction}

The accelerating shortage of fossil-based energy resources and shocks of oil prices, coupled with regulatory responses to impending global warming such as the climate change policy for reduction of greenhouse gas emissions, have prompted clearly the increasingly important sources of developing new and renewable energy such as wind, photovoltaic, thermal heat, and biological organisms (Kim etc., 2014). In recent years, the research and development $(\mathrm{R} \& \mathrm{D})$ expenditure on various renewable energy sources has increased aggressively. Many countries are aggressively investing in new and renewal energy R\&D programs and promoting policies to expand the use of renewable energy technologies. Indeed, R\&D can be seen as an important competitive tool to maintain the level of market profitability, as well as to ensure its anticipated return in the mid-term future for a science-based energy firm. Although R\&D can play a critical role for achieving new and renewable energy products, it undertakes huge and perilous uncertainties as the investment of R\&D has high risks and unknown returns, especially, the energy products may fail to market and the firm can not capture the emerging market opportunities (Hassanzadeh etc., 2012).

The R\&D decision-makers are faced with the main difficulties of increasing complexity and high expenditures. R\&D virtually involves a long and deferrable planning process and uncertain returns, which stems from several important features, for examples, capability implications, programmatic risks, total costs, resource allocations, and market return. Thus, these features have prompted decision-makers to continually search for more efficient 
R\&D investment decision-making methods which may lead to a better accommodation of inherent development difficulties. Therefore, decision-makers or policy-makers can use managerial flexibility to capture the peculiarities of policies, so as to accord with the initial policy targets.

Managerial flexibility means the utilization of management principles and approaches to adjust decision objects for contending with external circumstance changes, and to guarantee these methodologies to adapt the restrained terms (Lee \& Shih, 2010). The real option evaluation methodology can provide an analytical framework for managerial flexibility which can be neglected by the conventional analytical methods, for instances, discounted cash flow (DCF) and net present value (NPV). For example, Childs and Triantis (Childs \& Triantis, 1999) considered the real option valuation of R\&D programs under dynamic and uncertain environments, in which investment policies should balance the negative forces of incremental expenditures and the decrease of investment flexibility in allusion to the positive effects of fast uncertainty solutions and expedited cash flows. Huchzermeier and Loch (Huchzermeier \& Loch, 2001) examined five kinds of operational uncertainties, which include market returns, programme expenditures, development performance, demand levels, and development times. And they concluded that these variabilities may decrease the possibility of managerial flexibility. Krishnan and Bhattacharya (Krishnan $\&$ Bhattacharya, 2002) proposed that the "pizza-bin" method of abandoning potential technologies could not efficiently provide firms with profits, while the challenges to various products are huge. Santiago and Vakili (Santiago \& Vakili, 2005) considered the situation that whether the uncertainty increases the flexible value of R\&D project, which can be seen as the ability to carry new products into market with the least variabilities. They found the negative conclusions in contrast to Huchzermeier \& Loch (2001). Wang and Yang (Wang \& Yang, 2012) developed a flexibility management approach based on Huchzermeier \& Loch (2001) for capturing the option value of development projects. Bommel etc. (Bommel etc., 2014) presented a quantitative approach for determining the optimal ordering of operating R\&D projects drawing on the same underlying technology based on the real option analysis structure of Huchzermeier \& Loch (2001).

As mentioned above, common quantitative studies on managerial flexibility of $R \& D$ investment decision can be categorized as Black and Scholes equations, binominal lattice model, contingent claims analysis, and dynamic programming model (Wang \& Yang, 2012). Although the major quantitative formulations in these research are presented on account of statical information, many researchers strive to construct random models which can describe the natural attributes of R\&D investment decision. However, an obvious defect of these stochastic models is that the real data required for development projects are often difficult to collect effectively for the sake of absence for sufficient historical information. Therefore, randomly stemming from a proper probability distribution to describe $\mathrm{R} \& \mathrm{D}$ properties, for instances, market payoff, resource expenditure, or product performance, may be full of difficulties. Fuzzy set presented by Zadeh (1965) is a strict quantitative outline for describing vague and inaccurate messages in mentioned above situations. Fuzz theory provides an appropriate structure for dealing with imprecise and vague development attributions, when it is difficult to obtain enough data, or even to collect timely historical information. Fuzzy set can effectively present a precise description of subjective judgements. Furthermore, it can be particularly efficient on the condition that the feasible value scopes of development attributions may be provided by experts' subjective opinions.

The literature about fuzzy set applied to managerial flexibility of R\&D investment decision has been developed abundantly under incomplete information. As an early application, Carlsson and Fullér (Carlsson \& Fullér, 2003) formulated a simple fuzzy real option valuation framework in which the expected cash flows and costs are all described as trapezoidal fuzzy sets. In order to obtain the optimal exercise time, the possibilistic mean value and variance of fuzzy numbers are presented in a fuzzy environment. Wang and Hwang (Wang \& Hwang, 2007) developed a compound options valuation method which can be applied to estimate the values of development projects. Otherwise, the proposed methodology can be transformed to decision-making analysis assistants which can be used to select the suitable R\&D project under an uncertain circumstance. Liao and Ho (Liao \& Ho, 2010) presented a fuzzy binomial options model in which managerial flexibilities of R\&D projects can be revealed in vague and fuzzy situations. Furthermore, the fuzzy NPV of investment projects can be described by right-skewed possibility distribution in that managerial flexibilities maintain the potential of increasing return while restricting the negative loss.

An obvious shortcoming of the mentioned-above methodologies is the strict hypothesis of the option pricing models, because it is often supposed that product payoff follows the stochastic geometric Brownian motion. Realizing this restriction, Wang etc. (Wang etc., 2011) presented an improvement of Least Squares Monte-Carlo simulation used to the quantitative evaluations for fuzzy real option pricing of risky projects on the conditions of the sample paths generated by backward induction method. You etc. (You etc., 2012) adopted a fuzzy real option approach 
for enterprise resource planning (ERP) investment in which fuzzy returns evaluation is applied to dealing with uncertain attributes for minimizing the failure loss of ERP projects. Hassanzadeh etc. (Hassanzadeh etc., 2012) developed an effective $R \& D$ portfolio selection methodology in which the fuzzy pay-off model is used to valuate the flexible value of development project. They, moreover, discussed the value of managerial flexibility, that is not emphasized in the NPV-based evaluation framework.

This paper considers a multi-stages decision model for the optimal investment policy of energy R\&D project, taking into account $R \& D$ variabilities as risk events described by fuzzy variable that have a optimistic or pessimistic effects on the policy targets. It is always obvious that decision makers do not really prefer to handle complex mathematical formulations of decision-making. Therefore, simplicity should be taken into consideration for striving to maintain distinction to the greatest extent. Dynamic programming model, which is composed of uncertain $R \& D$ information and described as more realistic formulation of investment planing by mathematical equations, is proposed to analyze crucial development risks methodically and determine proper decision operations ahead of increasing managerial flexibility based on real option analysis in accordance with Santiago and Vakili (2005). Since managerial flexibility is not the internal attribute of energy R\&D project, the presented method can recognize the potential risky factors, develops a serious of real options to capture the risky factors, afterwards the fuzzy decision model is used to evaluate the value of opportunity and take the decision options, which can maximize the market payoff of R\&D project. R\&D decision makers can obtain managerial flexibility of risky energy R\&D project by improving its upside potential, and avoid threats by restricting negative losses with respect to the original prospection.

The organization of the paper is given as follows. In the next section, we review the fuzzy representation of uncertain and flexible information and the basics of fuzzy variables and quantitative credibility theory relevant to the methodology presented. In Section 3, the fuzzy multi-stages optimization framework for energy R\&D evaluation is developed. In Section 4, we build to derive the theoretical model and describe the value of managerial flexibility. Section 5 concludes this paper.

\section{Fuzzy Variables Preliminaries}

Zadeh (1965) first introduced the theory of fuzzy set, and applied it to deal with the uncertain sets without clear boundaries. For a more extensive application of fuzzy theory, Liu (2002) proposed fuzzy variables and credibility theory. Here, the basic definitions and operations of fuzzy variables and credibility theory will be reminded in the following.

\subsection{Fuzzy Variables and Credibility Theory}

Definition 1. (Nahmias, 1978) Assume that $\Theta$ is a nonempty set representing the sample space, and $\mathcal{P}(\Theta)$ is the power set of $\Theta$ (namely, $\mathcal{P}(\Theta)$ is the collection of all subsets of $\Theta$ ). For each $A \in \mathcal{P}(\Theta)$, a nonnegative number $\operatorname{Pos}\{A\}$ is named as the possibility measure that implies the occurring possibility of event $A$, so

(1) $\operatorname{Pos}\{\emptyset\}=0, \operatorname{Pos}(\Theta)=1$;

(2) $\operatorname{Pos}\left\{\bigcup_{k} A_{k}\right\}=\sup _{k} \operatorname{Pos}\left\{A_{k}\right\}$ for each collection $\left\{A_{k}\right\}$ in $\mathcal{P}(\Theta)$.

Then, the function Pos is defined as a possibility measure, such that the triplet $(\Theta, \mathcal{P}(\Theta)$, Pos) is said to be a possibility space.

Theorem 1. (Liu, 2002) Assume that $(\Theta, \mathcal{P}(\Theta)$, Pos) is a possibility space. We can obtain

(1) $0 \leq \operatorname{Pos}\{A\} \leq 1$ for any $A \in \mathcal{P}(\Theta)$;

(2) $\operatorname{Pos}\{A\} \leq \operatorname{Pos}\{B\}$ whenever $A \subset B$;

(3) $\operatorname{Pos}\{A \cup B\} \leq \operatorname{Pos}\{A\}+\operatorname{Pos}\{B\}$ for any $A, B \in \mathcal{P}(\Theta)$. It means that the possibility measure is sub-additive.

Definition 2. (Liu, 2002) A fuzzy variable $\xi$ can be named as a mapping function from the possibility space $(\Theta, \mathcal{P}(\Theta)$, Pos) to the set of real numbers $\mathcal{R}$.

Definition 3. (Liu, 2002) Suppose $\xi$ is a fuzzy variable on possibility space $(\Theta, \mathcal{P}(\Theta)$, Pos).

(a) If $\operatorname{Pos}\{\xi<0\}=0$, then $\xi$ is nonnegative.

(b) If $\operatorname{Pos}\{\xi \leq 0\}=0$, then $\xi$ is positive.

(c) If $\operatorname{Pos}\{\xi=x\}$ is a continuous function of $x$, then $\xi$ is continuous. 
Theorem 2. (Liu, 2002) If the fuzzy variable $\xi$ is continuous, then both $\operatorname{Pos}\{\xi \leq x\}$ and $\operatorname{Pos}\{\xi \geq x\}$ are continuous functions of $x$. Otherwise, $\operatorname{Pos}\{x \leq \xi \leq y\}$ is a continuous function on $\{(x, y) \mid x<y\}$.

Definition 4. (Liu, 2002) Suppose that $\xi$ is a fuzzy variable on possibility space $(\Theta, \mathcal{P}(\Theta)$, Pos), $x \in \mathcal{R}$. Then its membership function can be obtained through the possibility measure with

$$
\mu(x)=\operatorname{Pos}\{\theta \in \Theta \mid \xi(\theta)=x\} .
$$

Example 1. A triangular fuzzy variable can be described as the triplet $\left(r_{1}, r_{2}, r_{3}\right)$ with three crisp numbers $r_{1}<$ $r_{2}<r_{3}$, then its membership function is expressed by

$$
\mu(x)=\left\{\begin{array}{cl}
\frac{x-r_{1}}{r_{2}-r_{1}}, & \text { if } r_{1} \leq x \leq r_{2} \\
\frac{x-r_{3}}{r_{2}-r_{3}}, & \text { if } r_{2} \leq x \leq r_{3} \\
0, & \text { otherwise. }
\end{array}\right.
$$

Remark 1. In the real world, we can use a fuzzy variable $\eta$ to describe some uncertain information. Allow for that $\mu(x)$ signifies the occurring possibility of the event which $\eta$ equals to $x$ for a given real number $x$, then it may be applied to identify the magnitude of R\&D risk. For instance, the R\&D project risk caused by uncertain event with incomplete information may be characterised as a fuzzy variable. So $\mu(x)$ can means the occurring possibility which the realistic measure of risk is just equal to $x$.

Definition 5. (Liu, 2002) Let $\xi$ and $\eta$ be two fuzzy variables defined on the possibility space $(\Theta, \mathcal{P}(\Theta)$, Pos), then we say $\xi=\eta$ if and only if $\xi(\theta)=\eta(\theta)$ for all $\theta \in \Theta$.

Definition 6. (Liu, 2002) A series of fuzzy variables $\xi_{1}, \xi_{2}, \ldots, \xi_{n}$ are considered to be independent if and only if

$$
\operatorname{Pos}\left\{\xi_{i} \in B_{i}, i=1,2, \ldots, n\right\}=\min _{1 \leq i \leq n} \operatorname{Pos}\left\{\xi_{i} \in B_{i}\right\}
$$

for any Borel sets $B_{1}, B_{2}, \ldots, B_{n}$ of $\mathfrak{R}$.

Based on possibility measure Pos, we now recall necessity measure depicted by Nec and credibility measure depicted by Cr of fuzzy event with the following definitions.

Definition 7. (Liu, 2004) Assume that $(\Theta, \mathcal{P}(\Theta), P o s)$ is a possibility space, $A$ is a set in $\mathcal{P}(\Theta)$, and $A^{c}$ is the opposite set of A. So necessity measure of $A$ can be described as the impossibility of the opposite set $A^{c}$ with the following formulation

$$
\operatorname{Nec}\{A\}=1-\operatorname{Pos}\left\{A^{c}\right\} .
$$

Theorem 3. (Liu, 2004) Suppose that $(\Theta, \mathcal{P}(\Theta)$, Pos) is a possibility space. Then we have

(1) $\operatorname{Nec}\{\Theta\}=1, \operatorname{Nec}\{\emptyset\}=0$;

(2) $\operatorname{Nec}\{A\}=0$ whenever $\operatorname{Pos}\{A\}<1$;

(3) $\operatorname{Nec}\{A\} \leq \operatorname{Nec}\{B\}$ whenever $A \subset B$;

(4) $\operatorname{Nec}\{A\}+\operatorname{Pos}\left\{A^{c}\right\}=1$ for any $A \in \mathcal{P}(\Theta)$.

Definition 8. (Liu \& Liu, 2002) Assume that $(\Theta, \mathcal{P}(\Theta)$, Pos) is a possibility space, and A is a set in $\mathcal{P}(\Theta)$. It follows that credibility measure of $A$ can be depicted as the mean value of its possibility and necessity with the following formulation

$$
\operatorname{Cr}\{A\}=\frac{1}{2}(\operatorname{Pos}\{A\}+\operatorname{Nec}\{A\}) .
$$

Remark 2. From the described theory mentioned above, we know that even if its possibility is 1 , the fuzzy event still has the failure risk. On the other hand, even if its necessity is 0 , the fuzzy event still maybe holds. On the contrary, if its credibility is 1 , then the fuzzy event must holds. Otherwise, if its credibility is 0 , then the fuzzy event must fails. So credibility maybe plays the same role as probability measure.

Theorem 4. (Liu, 2002) Assume that $(\Theta, \mathcal{P}(\Theta)$, Pos) is a possibility space, and $A$ is a set in $\mathcal{P}(\Theta)$. Thus we show that

$$
\operatorname{Nec}\{A\} \leq \operatorname{Cr}\{A\} \leq \operatorname{Pos}\{A\}
$$


Theorem 5. (Liu, 2004) Assume that $(\Theta, \mathcal{P}(\Theta)$, Pos $)$ is a possibility space. Thus, it can be shown that

(1) $\operatorname{Cr}\{\Theta\}=1, \operatorname{Cr}\{\emptyset\}=0$;

(2) $\operatorname{Cr}\{A\} \leq \operatorname{Cr}\{B\}$ whenever $A \subset B$;

(3) $\mathrm{Cr}$ is self dual, namely, $\operatorname{Cr}\{A\}+\operatorname{Cr}\left\{A^{c}\right\}=1$ for any $A \in \mathcal{P}(\Theta)$;

(4) $\mathrm{Cr}$ is sub-additive, namely, $\operatorname{Cr}\{A \cup B\} \leq \operatorname{Cr}\{A\}+\operatorname{Cr}\{B\}$ for any $A, B \in \mathcal{P}(\Theta)$.

Definition 9. (Liu \& Liu, 2002) Assume that $\xi$ denotes a fuzzy variable. Then we show that the expected value of $\xi$ is given as follows

$$
E[\xi]=\int_{0}^{+\infty} \operatorname{Cr}\{\xi \geq r\} \mathrm{d} r-\int_{-\infty}^{0} \operatorname{Cr}\{\xi \leq r\} \mathrm{d} r
$$

on condition that at least one of the two integrals is finite.

Definition 10. (Liu, 2002) Suppose that $\xi$ is a fuzzy variable, and $\alpha \in(0,1]$. So we have the following critical values.

$$
\xi_{\text {inf }}(\alpha)=\inf \{r \mid \operatorname{Pos}\{\xi \leq r\} \geq \alpha\} \quad \text { and } \quad \xi_{\text {sup }}(\alpha)=\sup \{r \mid \operatorname{Pos}\{\xi \geq r\} \geq \alpha\}
$$

represent the $\alpha$-pessimistic value and the $\alpha$-optimistic value of $\xi$, respectively.

Proposition 1. (Liu \& Liu, 2002) Assume that $\xi$ is a fuzzy variable, and its expected value is denoted by E[ $\xi]$. Thus, we have the following equivalent expression of expected value.

$$
E[\xi]=\frac{1}{2} \int_{0}^{1}\left(\xi_{\text {inf }}(\alpha)+\xi_{\text {sup }}(\alpha)\right) \mathrm{d} \alpha .
$$

Definition 11. (Liu \& Liu, 2002) Assume that $\xi$ is a fuzzy variable, and its expected value can be denoted by a finite number $M$. Then we have the variance of $\xi$ as follows

$$
V[\xi]=E\left[(\xi-M)^{2}\right]
$$

\subsection{Fuzzy Arithmetic}

Definition 12. (Liu, 2002) We suppose that $f: \mathfrak{R}^{n} \rightarrow \mathfrak{R}$ is a mapping function, and $\xi_{1}, \xi_{2}, \ldots, \xi_{n}$ are fuzzy variables on the possibility space $\left(\Theta, \mathcal{P}(\Theta)\right.$, Pos). Then we obtain $\xi=f\left(\xi_{1}, \xi_{2}, \ldots, \xi_{n}\right)$ is a fuzzy variable defined as

$$
\xi(\theta)=f\left(\xi_{1}(\theta), \xi_{2}(\theta), \ldots, \xi_{n}(\theta)\right)
$$

for any $\theta \in \Theta$.

Example 2. Let $\xi_{1}$ and $\xi_{2}$ be two fuzzy variables on the possibility space $(\Theta, \mathcal{P}(\Theta)$, Pos). Then their sum and product are expressed by

$$
\left(\xi_{1}+\xi_{2}\right)(\theta)=\xi_{1}(\theta)+\xi_{2}(\theta),\left(\xi_{1} \times \xi_{2}\right)(\theta)=\xi_{1}(\theta) \times \xi_{2}(\theta)
$$

for any $\theta \in \Theta$.

Theorem 6. (Liu \& Liu, 2002) Let $\xi$ and $\eta$ be independent fuzzy variables with finite expected values, a and $b$ are any two real numbers. Thus,

$$
E[a \xi+b \eta]=a E[\xi]+b E[\eta]
$$

Theorem 7. (Liu \& Liu, 2002) Assume that $\xi$ is a fuzzy variables with a finite variance, and a, $b$ are two real numbers, so

$$
V[a \xi+b]=a^{2} V[\xi]
$$

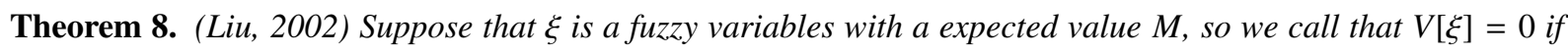
and only if $\operatorname{Cr}\{\xi=M\}=1$. 


\section{Multi-stages Decision Model for Energy R\&D Project}

The energy technology development is often involved with some types of risk factors, especially technical and market risks, long and deferrable planning duration, and uncertain final payoffs. The R\&D process may usually be described as a multi-stages decision process in which a real option structure is recognized to deal with the identified potential risks, and the proper options which can increase the potential value of managerial flexibility for energy R\&D project are evaluated and adopted (Lee \& Shih, 2010).

\subsection{Performance State and Management Decision}

In this study, we adopt the real option structure in accordance with Huchzermeier \& Loch (2001) and Santiago \& Vakili (2005). The interested readers may consult the detail modelling methods in these literature. We suppose that the R\&D project of new and renewable energy has $T$ development stages: $t=0,1, \cdots, T-1$. For any stage $t$, the decision maker considers three kinds of options: the option to continue energy development, the option to improve energy development, and the option to abandon energy development. In that the project's value depends on the final product introduced to energy market, the product value can be represented by the performance state of $\mathrm{R} \& \mathrm{D}$ project. It is assumed that $X_{t}$ denotes the development performance of $\mathrm{R} \& \mathrm{D}$ project at the starting point of stage $t$, and $X_{0}=0$. We assume that fuzzy variable $\xi_{t}$ depicts the uncertain description of development risk at stage $t$, and $u_{t}$ represents the management decision at the starting point of stage $t$. Thus, the development status of R\&D project at the completion of stage $t$ can be described as

$$
X_{t+1}=\left\{\begin{array}{cl}
\mathrm{X}_{t}+k\left(u_{t}\right)+\xi_{t}, & \text { if } u_{t}=\text { continue or improve, } \\
X_{t}, & \text { if } u_{t}=\text { abandon, }
\end{array}\right.
$$

where,

$$
k\left(u_{t}\right)= \begin{cases}0, & \text { if } u_{t}=\text { continue } \\ 1, & \text { if } u_{t}=\text { improve }\end{cases}
$$

Consequently, if the energy development project is undertaken at the "continue" level under the increasing oil price, the performance at the following period will maintain the same value as that at present stage add some uncertain measure. Otherwise, if the project is set up on the "improve" level under the higher and higher oil price, it takes the value that one unit of improvement add the uncertain effect. Otherwise, if the project is funded at the "abandon" level for some reason, the development performance remains in the stopped state in the following stages of the project.

To describe the proposition about performance level of energy R\&D project, the ranking criteria of fuzzy variables can be given as follows.

Definition 13. (Liu, 2002) Let $\xi$ and $\eta$ be two fuzzy variables defined on the possibility space $(\Theta, \mathcal{P}(\Theta)$, Pos). We say $\xi \geq \eta$ if and only if $\xi(\theta) \geq \eta(\theta)$ for all $\theta \in \Theta$. That is, $\xi \geq \eta$ if and only if $\Phi(x) \leq \Psi(x)$ for all $x \in \mathfrak{R}$, where $\Phi$ and $\Psi$ are credibility distributions of $\xi$ and $\eta$, respectively.

To concentrate on the implementation issue, four ranking criteria for ranking fuzzy variables are given by Liu (2002) as follows.

(1) Expected Value Criterion. It can be said that $\xi>\eta$ if and only if $E[\xi]>E[\eta]$, where $E$ denotes the expected value formulation of fuzzy variable.

(2) Optimistic Value Criterion. It can be said that $\xi>\eta$ when and only when, for certain given confidence level $\alpha \in(0,1]$, then $\xi_{\text {sup }}(\alpha)>\eta_{\text {sup }}(\alpha)$, in which $\xi_{\text {sup }}(\alpha)$ and $\eta_{\text {sup }}(\alpha)$ are the $\alpha$-optimistic values of $\xi$ and $\eta$, respectively.

(3) Pessimistic Value Criterion. It can be said that $\xi>\eta$ when and only when, for certain given confidence level $\alpha \in(0,1]$, then $\xi_{\text {inf }}(\alpha)>\eta_{\text {inf }}(\alpha)$, in which $\xi_{\text {inf }}(\alpha)$ and $\eta_{\text {inf }}(\alpha)$ are the $\alpha$-pessimistic values of $\xi$ and $\eta$, respectively.

(4) Credibility Criterion. It can be said that $\xi>\eta$ when and only when $\operatorname{Cr}\{\xi \geq \bar{r}\}>\operatorname{Cr}\{\eta \geq \bar{r}\}$ for certain given level $\bar{r}$. 
In the following, we give the proposition of the performance state of energy R\&D project under fuzzy environments.

Proposition 2. We consider an RED project with two decision situations. In situation 1, the RED project is supposed that either to continue or to improve; in situation 2, it is supposed that to continue at all stages (namely, the second situation accords with the assumption of traditional NPV calculations). Assume that $X_{t}$ and $X_{t}^{\prime}$ are the states of the project under situation 1 and 2 at the beginning of stage $t$, respectively. So we have

$$
X_{t} \geq X_{t}^{\prime}
$$

Proof. We assume that the initial performances of the project are equal at stage 0, i.e., $X_{0}=X_{0}^{\prime}=0$. Otherwise, $X_{t}$ and $X_{t}^{\prime}$ are fuzzy variables on the possibility space $\left(\Theta, \mathcal{P}(\Theta)\right.$, Pos). Assume that $X_{t-1}(\theta) \geq X_{t-1}^{\prime}(\theta)$ for all $\theta \in \Theta$ at the stage of $(t-1)$. Furthermore, the optimal decision $u^{*}$ are taken by decision maker at the stage of $(t-1)$ in scenario 1 . Then the performance of stage $t$ can be given as follows.

$$
X_{t}(\theta)=X_{t-1}(\theta)+k\left(u^{*}\right)+\xi_{t-1}(\theta) \geq X_{t-1}^{\prime}(\theta)+\xi_{t-1}(\theta)=X_{t}^{\prime}(\theta) .
$$

The proof is completed.

\subsection{Development Uncertainty}

The uncertainty of new and renewable energy technology development $\xi_{t}(t=0, \ldots, T-1)$ contains not only the internal technology risk, but also the external market risk sources. Huchzermeier \& Loch (2001) described five kinds of uncertain factors, i.e., market return variability, expenditure variability, performance variability, market requirement variability, and schedule variability. Here, the $\xi_{t}$ are described as a set of independent and identically distributed (iid) bounded fuzzy variables defined on the possibility spaces $(\Theta, \mathcal{P}(\Theta)$, Pos). Furthermore, the expected values of $\xi_{t}$ are assumed as 0 because that the project performance may take on improvement or deterioration state in face of different uncertain events. The expected value operator and variance operator of $\xi_{t}$ are given as the following formulations

$$
\begin{gathered}
E\left[\xi_{t}\right]=\int_{0}^{+\infty} \operatorname{Cr}\left\{\xi_{t} \geq r\right\} \mathrm{d} r-\int_{-\infty}^{0} \operatorname{Cr}\left\{\xi_{t} \leq r\right\} \mathrm{d} r=0, \\
V\left[\xi_{t}\right]=E\left[\left(\xi_{t}-E\left[\xi_{t}\right]\right)^{2}\right] .
\end{gathered}
$$

Here, the variance $V\left[\xi_{t}\right]$ may be considered as a measure of development risk or variability.

In the methodology mentioned above, the value of development status can vary with the resolution of uncertainty. For instance, we assume that the development status of R\&D project is denoted by $X_{t}$ at the starting point of stage $t$, if the uncertainty presented by $\xi_{t}$ can be resolved, then we can obtain the new development status described as $X_{t}+k\left(u_{t}\right)+\xi_{t}$ when the stage $t$ is completed. Otherwise, all other factors of R\&D project maintain still the old status. Thus, it is said that the internal development uncertainty can be characterised by fuzzy variable $\xi_{t}$ at stage $t$. On the other hand, when we allow the development status involving the project performance about external market information, then the fuzzy variable $\xi_{t}$ can also reflect somewhat information of external market requirement in some sense. For instance, when the new market information represents a higher customers requirement level than before obtained, it can be reflected by a lower project performance status in the end of stage $t$.

\subsection{Development Cost and Market Payoff}

In this settings, the development cost can be effected by managerial flexibility through the decision choice of decision maker. When the decision maker chooses the abandon option, the development process of energy R\&D project may be timely stopped, hence there is no more development expenditure which will be employed. Otherwise, when the decision maker chooses other options, the incurred development cost can often be influenced by the following factors, such as the performance status at the review stage $t$, and the decision action chosen by decision maker in stage $t$. The cost function can be depicted by $C_{t}\left(u_{t}\right)$ as follows

$$
C_{t}\left(u_{t}\right)=\left\{\begin{array}{cl}
c(t), & \text { if } u_{t}=\text { continue } \\
c(t)+a(t), & \text { if } u_{t}=\text { improve } \\
0, & \text { if } u_{t}=\text { abandon }
\end{array}\right.
$$


Here, $c(t)$ is a continuation cost when the decision action is continuation, and $a(t)$ means an additional improvement cost with the exception of continuation cost $c(t)$ under the improvement option. Otherwise, there is no costs incurred at the stopped state.

The continuation cost $c(t)$ is a normal investment in order to maintain the project, but the improvement cost $a(t)$ is an extra cost for technological difficulties, i.e., increasing the $R \& D$ engineers or buying the experiment devices. It is need an original investment $I$ to carry out the energy R\&D project at the starting stage 0 . It is assumed that the investment is incurred at the beginning of each stage and the payoff or cost can be discounted with the riskfree discount factor $r$. At the final stage $T$, the developed product will be launched into the market. If the final performance status of developed product is denoted by $X_{T}$, then we can obtain a market payoff $R\left(X_{T}\right)$ with the following formulation

$$
R\left(X_{T}\right)= \begin{cases}\mathrm{m}, & \text { if } X_{T}<\eta, \\ M, & \text { if } X_{T} \geq \eta,\end{cases}
$$

where $\eta$ is a certain performance level required by market to satisfy the customers.

If the new and renewable energy product reaches or exceeds the market requirement level $\eta$, the energy firm can capture a large market return $M$. Then, the developed product will take on a competitive advantage over the same type of other product. Otherwise, when the developed product dose not meet the market requirement level $\eta$, the firm may only obtain a small market return $m$. It means that the product must to compete with other competitive product by the price strategy. Furthermore, we usually do not know the market requirement level $\eta$ before the developed product is commercialized. The market requirement level $\eta$ can be described as a fuzzy variable with the following expected value and variance

$$
\begin{gathered}
E[\eta]=\int_{0}^{+\infty} \operatorname{Cr}\{\eta \geq r\} \mathrm{d} r-\int_{-\infty}^{0} \operatorname{Cr}\{\eta \leq r\} \mathrm{d} r, \\
V[\eta]=E\left[(\eta-E[\eta])^{2}\right] .
\end{gathered}
$$

For any $\theta \in \Theta$, the development performance at stage $T$ can be expressed by $X_{T}(\theta)$, and $\operatorname{Cr}\left\{X_{T}(\theta) \geq \eta\right\}$ means the credibility of event $\left\{X_{T}(\theta) \geq \eta\right\}$. Then, the expected value of market payoff $R\left(X_{T}\right)$ can be given as follows.

$$
\begin{aligned}
E\left[R\left(X_{T}(\theta)\right)\right] & =\int_{0}^{+\infty} \operatorname{Cr}\left\{R\left(X_{T}(\theta)\right) \geq r\right\} \mathrm{d} r \\
& =\int_{0}^{m} \operatorname{Cr}\left\{R\left(X_{T}(\theta)\right) \geq r\right\} \mathrm{d} r+\int_{m}^{M} \operatorname{Cr}\left\{R\left(X_{T}(\theta)\right) \geq r\right\} \mathrm{d} r \\
& =m+\int_{m}^{M} \operatorname{Cr}\left\{X_{T}(\theta) \geq \eta\right\} \mathrm{d} r \\
& =m+\operatorname{Cr}\left\{X_{T}(\theta) \geq \eta\right\} \cdot \int_{m}^{M} \mathrm{~d} r \\
& =m+\operatorname{Cr}\left\{X_{T}(\theta) \geq \eta\right\} \cdot(M-m) .
\end{aligned}
$$

The credibility distribution function of fuzzy variable $\eta$ is assumed as $\Phi(\cdot)$. Then

$$
E\left[R\left(X_{T}(\theta)\right)\right]=m+\Phi\left(X_{T}\right) \cdot(M-m) .
$$

\subsection{Value Function and Optimal Policy}

Following the traditional dynamic programming structure of multi-stages decision problems, the decision choice of intermediate stage can be seen as a judgement of whether the devoted cost of this stage is fully recovered by the final market return. The midterm expenditure with each decision choice can be determined through the cost function equation (17) mentioned above; thus, each decision choices may be dependent on its assessment for the final market return, in which the value function can represent the measurement of this assessment. Therefore, the features of value function can greatly influence the optimal choices. In particular, the internal associativity can be characterized by the standard dynamic programming with the following formulation

$$
V_{t}\left(X_{t}\right)=\max _{u_{t}} E\left[-C_{t}\left(u_{t}\right)+\frac{1}{1+r} V_{t+1}\left(X_{t+1}\left(X_{t}, u_{t}, \xi_{t}\right)\right)\right] .
$$


The value function $V_{t}\left(X_{t}\right)$ means the expected discounted value of performance status at the starting point of stage $t$. For the final stage $T, V_{T}\left(X_{T}\right)=E\left[R\left(X_{T}\right)\right]$. The $V_{t}\left(X_{t}\right)$ is the expected discounted net return under the optimal policy. So the investment before stage $t$ can be seen as the sunk cost. The decision maker only consider the comparison of the cost and payoff at stage $t$. Thus, the type of value function is a critical factor for obtaining the optimal policy through the traditional backward recursion. Santiago \& Vakili (2005) gave the proposition of value function under random environment. Now, we describe the proposition under fuzzy uncertainty.

Proposition 3. Let $x$ be the performance state of the project. If the expected value of final payoff function $E[R(x)]$ is a monotone nondecreasing function, so the value function of any reviewed stage, i.e., $V_{t}(x), t=0,1, \ldots, T-1$, is also a monotone non-decreasing function.

Proof. The proof can be operated by the standard backward recursion. $V_{T}(x)=E[R(x)]$ is monotone nondecreasing in $x$ by assumption. Now we assume that $V_{t+1}(x)$ is monotone nondecreasing in $x$. Let $x_{1}$ and $x_{2}$ be two performance states at stage $t$, and $x_{2}>x_{1}$. Then we need to prove that $V_{t}\left(x_{2}\right) \geq V_{t}\left(x_{1}\right)$ only.

Choosing the optimal decision $u^{*}$ to maximize the value function $V_{t}\left(x_{1}\right)$ when the performance state is $x_{1}$ at stage $t$. Assume that the decision choice is also $u^{*}$ when the performance state is $x_{2}$ at stage $t$. Here, the firm obtains the value function $V_{t}^{\prime}\left(x_{2}\right)$ which maybe is not the maximum under this situation. Then we have

(1) When the optimal decision $u^{*}$ is continue or improve, it can be shown that

$$
V_{t}^{\prime}\left(x_{2}\right)-V_{t}\left(x_{1}\right)=\frac{1}{1+r} E\left[V_{t+1}\left(x_{2}+k\left(u^{*}\right)+\xi_{t}\right)-V_{t+1}\left(x_{1}+k\left(u^{*}\right)+\xi_{t}\right)\right] .
$$

(2) When the optimal decision $u^{*}$ is abandon, then

$$
V_{t}^{\prime}\left(x_{2}\right)-V_{t}\left(x_{1}\right)=0
$$

Assume that $\xi_{t}$ is a fuzzy variable defined on the possibility space $(\Theta, \mathcal{P}(\Theta)$, Pos $)$, so $x_{1}+k\left(u^{*}\right)+\xi_{t}$ and $x_{2}+k\left(u^{*}\right)+\xi_{t}$ are all fuzzy variables on the possibility space $\left(\Theta, \mathcal{P}(\Theta)\right.$, Pos). Since $x_{2}>x_{1}$, then $x_{2}+k\left(u^{*}\right)+\xi_{t}>x_{1}+k\left(u^{*}\right)+\xi_{t}$. Hence, it follows that

$$
V_{t+1}\left(x_{2}+k\left(u^{*}\right)+\xi_{t}\right)-V_{t+1}\left(x_{1}+k\left(u^{*}\right)+\xi_{t}\right) \geq 0 .
$$

Here, the fuzzy variable is nonnegative, so the expected value of fuzzy variable is also nonnegative. Hence,

$$
E\left[V_{t+1}\left(x_{2}+k\left(u^{*}\right)+\xi_{t}\right)-V_{t+1}\left(x_{1}+k\left(u^{*}\right)+\xi_{t}\right)\right] \geq 0 .
$$

and so,

$$
V_{t}^{\prime}\left(x_{2}\right)-V_{t}\left(x_{1}\right) \geq 0
$$

Since $V_{t}\left(x_{2}\right)$ is the maximal project value under the optimal decision at state $x_{2}$, it follows that $V_{t}\left(x_{2}\right) \geq V_{t}^{\prime}\left(x_{2}\right)$. Hence, we obtain that $V_{t}\left(x_{2}\right)-V_{t}\left(x_{1}\right) \geq V_{t}^{\prime}\left(x_{2}\right)-V_{t}\left(x_{1}\right) \geq 0$. That is,

$$
V_{t}\left(x_{2}\right) \geq V_{t}\left(x_{1}\right),
$$

and the proof is completed.

By making use of the monotonicity of value function, we then obtain the following lemma of abandon decision.

Lemma 1. If the optimal decision is to abandon an energy $R \mathcal{E} D$ project with performance status $x$ at stage $t$, then at any status smaller than $x$ at this stage, the optimal decision must be to abandon the energy RED project.

Proof. Let $x^{\prime}$ be an other performance status at stage $t$, and $x^{\prime}<x$. Consider that the optimal decision is to abandon the energy R\&D project with the status $x$ at stage $t, V_{t}(x)=0$. Monotonicity of $V(x)$ means that $V_{t}\left(x^{\prime}\right) \leq V_{t}(x)$. Since $V_{t}(x)=0$, we see that $V_{t}\left(x^{\prime}\right) \leq 0$. Otherwise, $V_{t}\left(x^{\prime}\right) \geq 0$ since terminating the energy R\&D project at performance status $x^{\prime}$ generates a value of 0 . Thus, $V_{t}\left(x^{\prime}\right)=0$. We can know that the optimal decision is to abandon the energy R\&D project with status $x^{\prime}$ at stage $t$.

\section{Value of Management Flexibility for Energy R\&D Project}

Huchzermeier \& Loch (2001) presented five kinds of drivers characterized by uncertainty, in which the market factors include market return variability and market requirements variability, the project factors are composed of 
three parts, namely, budget variability, performance variability, and schedule variability. Here, we only focus on three uncertain factors, including market payoff, performance state, and market requirement, as the analysis in the framework of dynamic programming model which is based on the standard structure of Santiago \& Vakili (2005). Therefore, the budget and schedule are treated as deterministic factors and the other uncertain factors can be characterized as fuzzy variables which more accords with real situations. In addition, the parameters according to these variabilities are the difference of market payoff $(M-m)$, the variance of development uncertainty $V\left[\xi_{t}\right]$, and the variance of market requirement level $V[\eta]$. As the development uncertainty is mainly characterized by the market payoff, this paper only analyzes the market factors which contains the market payoff and the market requirement.

\subsection{Increased Variability of Market Payoff on the Option Value}

For the influence of market payoff, we would first debate the optimistic sides of the effect of increased variability. In the above section, we suppose that the market payoff may take on two potential outcomes. When the final product performance $X_{T}$ is larger than the customer requirement level $\eta$, then the firm can obtain the premium market payoff $M$. On the other hand, when the final product performance $X_{T}$ is smaller than the customer requirement level $\eta$, then the firm can obtain the basic market payoff $m$. The measure of variability for market payoff may be denoted by the range $(M-m)$. Let $b=(M+m) / 2$ and $b=(M-m) / 2$ denotes the mean value and risk magnitude of market payoff, respectively. Then $M=b+d$ and $m=b-d$.

Consider two projects that are identical except for their market payoff. The payoff values for project 1 are denoted by $M$ and $m$ and those for project 2 by $M^{\prime}$ and $m^{\prime}$. Suppose these two projects have the same mean value of market payoff $b=(M+m) / 2=\left(M^{\prime}+m^{\prime}\right) / 2$. The different risk magnitudes of market payoff are $d$ and $d^{\prime}$, respectively, and $d^{\prime}>d$. The market payoff function for project 1 is represented as $R(x)$, and $R^{\prime}(x)$ denotes the market payoff function for project 2 , where $x$ is the performance status of energy R\&D project. Therefore, the theory of expected payoff can be given as follows.

Theorem 9. The difference of expected market payoff $\left(E\left[R^{\prime}(x)\right]-E[R(x)]\right)$ is an increasing function of $x$.

Proof. Let the market requirement level $\eta$ be a fuzzy variable on the possibility space $(\Theta, \mathcal{P}(\Theta)$, Pos), which has the credibility function $\Phi(x)$. Then

$$
\Phi(x)=\operatorname{Cr}\{\theta \in \Theta \mid \eta(\theta) \leq x\} .
$$

Following the equation (22), we have

$$
E[R(x)]=m+\Phi(x) \cdot(M-m)=b-d+2 d \cdot \Phi(x)=b+d(2 \Phi(x)-1)
$$

and

$$
E\left[R^{\prime}(x)\right]=m^{\prime}+\Phi(x) \cdot\left(M^{\prime}-m^{\prime}\right)=b-d^{\prime}+2 d^{\prime} \cdot \Phi(x)=b+d^{\prime}(2 \Phi(x)-1) .
$$

The difference of these two functions is

$$
E\left[R^{\prime}(x)\right]-E[R(x)]=b+d^{\prime}(2 \Phi(x)-1)-(b+d(2 \Phi(x)-1))=\left(d^{\prime}-d\right)(2 \Phi(x)-1) .
$$

Here, $\Phi(x)$ is an increasing function of $x$, and $d^{\prime}-d>0$, then $E\left[R^{\prime}(x)\right]-E[R(x)]$ is also an increasing function of $x$. The proof is completed.

The value of R\&D project have two parts: the option value and the NPV (Net Present Value). So the option value can be denoted by the difference of project value and NPV. In fact, the NPV is just the project value when the decision is continue at all periods. Assume that the decision maker has not choose the abandon decision as the optimal policy, then the proposition can be described as follows.

Proposition 4. If the variability of market payoff increases, then the option value of energy RED project will increase.

Proof. Let $X_{t}$ be the performance status of project 1 at stage $t$ under the optimal policy, and let $X_{t}^{\prime}$ be the performance status under the NPV assumption. Otherwise, the value functions of project 2 are denoted by $V^{\prime}$ and $\bar{V}^{\prime}$ under the optimal policy and the same policy with project 1, respectively. It should be noted that these two projects are identical except for their market payoff. Then the project 2 has the same performance statuses $X_{t}$ and $X_{t}^{\prime}$ with 
project 1 . Hence, these two projects have the same development cost. The difference of value between project 1 and 2 at stage 0 under the optimal policy is given as follows.

$$
\bar{V}_{0}^{\prime}-V_{0}=\frac{E\left[R^{\prime}\left(X_{T}\right)\right]}{(1+r)^{T}}-\frac{E\left[R\left(X_{T}\right)\right]}{(1+r)^{T}}=\frac{E\left[R^{\prime}\left(X_{T}\right)\right]-E\left[R\left(X_{T}\right)\right]}{(1+r)^{T}} .
$$

Therefore, the subtraction of value under the NPV assumption is such that

$$
N P V_{0}^{\prime}-N P V_{0}=\frac{E\left[R^{\prime}\left(X_{T}^{\prime}\right)\right]}{(1+r)^{T}}-\frac{E\left[R\left(X_{T}^{\prime}\right)\right]}{(1+r)^{T}}=\frac{E\left[R^{\prime}\left(X_{T}^{\prime}\right)\right]-E\left[R\left(X_{T}^{\prime}\right)\right]}{(1+r)^{T}} .
$$

From Theorem 9, we see that the difference of expected market payoff is an increasing function and $X_{T} \geq X_{T}^{\prime}$ (from Proposition 2). Then

$$
\bar{V}_{0}^{\prime}-V_{0} \geq N P V_{0}^{\prime}-N P V_{0} .
$$

Since $V^{\prime} \geq \bar{V}^{\prime}$ under the optimal policy for project 2, it follows that

$$
V_{0}^{\prime}-V_{0} \geq \bar{V}_{0}^{\prime}-V_{0} \geq N P V_{0}^{\prime}-N P V_{0} .
$$

That is,

$$
V_{0}^{\prime}-N P V_{0}^{\prime} \geq V_{0}-N P V_{0} .
$$

Namely, the option value of project 2 is larger than or equal to the option value of project 1 . Thus the proof is completed.

\subsection{Increased Variability of Market Requirement on the Option Value}

The variability of market requirement can be measured by the variance, $V[\eta]$, of the fuzzy variable of market requirement level $\eta$. Furthermore, the expected value $E[\eta]$ holds constantly. Note that an increase of the variance $V[\eta]$ means a decrease of the known information about external market requirement at the time of product commercialized. The following proposition providers the influence on the option value by the variability of market requirement.

Proposition 5. If the variability of market requirement increases, then the option value will decrease.

Proof. Let the market requirement levels of project 1 and 2 be $\eta$ and $\eta^{\prime}$, respectively. Noted that $E[\eta]=E\left[\eta^{\prime}\right]$ and $V[\eta]<V\left[\eta^{\prime}\right]$. The other conditions are identical for these two projects. From equation (22), we see that the expected values of market payoff are equal for these two projects, and the variability of market payoff for project 2 is smaller than project 1 . That is,

$$
R^{\prime}\left(X_{T}\right)-R^{\prime}\left(X_{T-1}\right)<R\left(X_{T}\right)-R\left(X_{T-1}\right) .
$$

Following Proposition 4, we can obtain that the option value of project 2 is smaller than the option value of project 1 and the proof is thus completed.

\section{Conclusions}

This paper considers a simple multi-stages decision model for energy R\&D project, where the uncertain factors include market payoff, product performance, and market requirement level. The decision maker often faces three choice option: continue, improve, and abandon. Thus the management decisions of energy R\&D project have the value of managerial flexibility. We analysis the influence of market variability on the real option value. Specifically, the increased variability of market payoff can increase the option value of energy R\&D project; otherwise, the increased variability of market requirement can decrease the option value of energy $R \& D$ project. The outcomes of this research throw light on the work of energy $R \& D$ policy decision-makers. For example, the variabilities of product performance and market requirement are often little for a relatively mature product. The decision-maker can divides the few decision stages in that the project has the larger flexibility value. On the other hand, the decision-maker should schedules the many decision stages in order to obtain the larger flexibility value for the new and renewable energy which has the larger variabilities of product performance and market requirement.

\section{Acknowledgements}

The authors would like to acknowledge the financial support of the Humanities and Social Sciences Foundation of the Ministry of Education (10YJC630352), the Humanities and Social Sciences Major Program of Anhui Province 
(SK2014ZD018), and the Jinglong Company Research Project (RD13210009). The authors are sincerely grateful to the anonymous referees for helpful comments and suggestions.

\section{References}

Bommel, T., Mahieu, R., \& Nijssen, E. (2014). Technology trajectories and the selection of optimal R\&D project sequences. IEEE Transactions on Engineering Management, 61(4), 669-680. http://dx.doi.org/10.1109/TEM.2014.2349554

Carlsson, C., \& Fullér, R. (2003). A fuzzy approach to real option valuation. Fuzzy Sets and Systems, 139(2), 297-312. http://dx.doi.org/10.1016/S0165-0114(02)00591-2

Childs, P., \& Triantis, A. (1999). Dynamic R\&D investment policies. Management Science, 45(10), 1359-1377. http://dx.doi.org/10.1287/mnsc.45.10.1359

Hassanzadeh, F., Collan, M., \& Modarres, M. (2012). A practical approach to R\&D portfolio Selection using the fuzzy pay-Off method. IEEE Transactions on Fuzzy Systems, 20(4), 615-622. http://dx.doi.org/10.1109/TFUZZ. 2011.2180380

Huchzermeier, A., \& Loch, C. (2001). Project management under risk: using the real options approach to evaluate flexibility in R\&D. Management Science, 47(1), 85-101. http://dx.doi.org/10.1287/mnsc.47.1.85.10661

Kim, K., Lee, D., \& Park, S. (2014). Evaluation of R\&D investments in wind power in Korea using real option. Renewable and Sustainable Energy Reviews, 40, 335-347. http://dx.doi.org/10.1016/j.rser.2014.07.165

Krishnan, V., \& Bhattacharya, S. (2002). Technology selection and commitment in new product development: the role of uncertainty and design flexibility. Management Science, 48(3), 313-327. http://dx.doi.org/10.1287/mnsc.48.3.313.7728

Lee, S., \& Shih, L. (2010). Renewable energy policy evaluation using real option model $\nmid$ The case of Taiwan. Energy Economics, 32(S1), S67-S78. http://dx.doi.org/10.1016/j.eneco.2010.04.010

Liao, S., \& Ho, S. (2010). Investment project valuation based on a fuzzy binomial approach. Information Sciences, 180(11), 2124-2133. http://dx.doi.org/10.1016/j.ins.2010.02.012

Liu, B. (2002). Theory and Practice of Uncertain Programming. Heidelberg: Phisica-Verlag.

Liu, B. (2004). Uncertainty Theory: An Introduction to Its Axiomatic Foundations. Berlin: Springer-Verlag.

Liu, B., \& Liu, Y. (2002). Expected Value of Fuzzy Variable and Fuzzy Expected Value Models. IEEE Transactions on Fuzzy Systems, 10(4), 445-450. http://dx.doi.org/10.1109/TFUZZ.2002.800692

Nahmias, S. (1978). Fuzzy variables. Fuzzy Sets and Systems, 1(2), 97-110. http://dx.doi.org/10.1016/01650114(78)90011-8

Santiago, L., \& Vakili, P. (2005). On the value of flexibility in R\&D projects. Management Science, 51(8), 12061218. http://dx.doi.org/10.1287/mnsc. 1050.0387

Wang, J., \& Hwang, W. (2007). A fuzzy set approach for R\&D portfolio selection using a real options valuation model. Omega, 35(3), 247-257. http://dx.doi.org/10.1016/j.omega.2005.06.002

Wang, J., \& Yang, C. (2012). Flexibility planning for managing R\&D projects under risk. International Journal of Production Economics, 135(2), 823-831. http://dx.doi.org/10.1016/j.ijpe.2011.10.020

Wang, Q., Kilgour, D., \& Hipel, K. (2011). Fuzzy real options for risky project evaluation using least squares monte-carlo simulation. IEEE Systems Journal, 5(3), 385-395. http://dx.doi.org/10.1109/jsyst.2011.2158687

You, C., Lee, C., Chen, S., \& Jiao, R. (2012). A real option theoretic fuzzy evaluation model for enterprise resource planning investment. Journal of Engineering and Technology Management, 29(1), 47-61.

http://dx.doi.org/10.1016/j. jengtecman.2011.09.005

Zadeh, L. (1965). Fuzzy Sets. Information and Control, 8(3), 338-353.

\section{Copyrights}

Copyright for this article is retained by the author(s), with first publication rights granted to the journal.

This is an open-access article distributed under the terms and conditions of the Creative Commons Attribution license (http://creativecommons.org/licenses/by/3.0/). 